\title{
ON SOME FIXED POINT THEOREMS FOR MAPPINGS WITH GENERALIZED LIPSCHITZIAN ITERATES
}

\section{BALWANT SINGH THAKUR, BIRENDRA KUMAR SHARMA AND JONG SOO JUNG}

\begin{abstract}
In this paper we prove the following theorem: Let $p>1$ and let $E$ be a $p$-uniformly convex Banach space, $K$ a nonempty bounded closed convex subset of $E$, and $A=\left[t_{n, k}\right]_{n, k \geq 1}$ a strongly ergodic matrix. Let $T: K \rightarrow K$ be a continuous mapping satisfying: for each $x, y$ in $K$ and $i=1,2, \ldots$.
\end{abstract}

$$
\left\|T^{i} x-T^{i} y\right\| \leq a_{i}\|x-y\|+b_{i}\left(\left\|x-T^{i} x\right\|+\left\|y-T^{i} y\right\|\right)+c_{i}\left(\left\|x-T^{i} y\right\|+\left\|y-T^{i} x\right\|\right)
$$

where $a_{i}, b_{i}, c_{i}$ are nonnegative, $3 b_{i}+3 c_{i} \leq 1$, and

$$
\liminf _{n \rightarrow \infty} \inf _{m=0,1,2, \ldots} \sum_{k=1}^{\infty} t_{n, k} \cdot 2^{p-1}\left\{\alpha_{k+m}^{p}+\beta_{k+m}^{p}\right\}<1+c_{p}
$$

where $\alpha_{i}=\frac{a_{i}+b_{i}+c_{i}}{1-b_{i}-c_{i}}, \beta_{i}=\frac{2 b_{i}+2 c_{i}}{1-b_{i}-c_{i}}$, and $c_{p}>0$ is some constant. Then $T$ has a fixed point in $K$.

\section{Introduction and Preliminaries}

Let $K$ be a nonempty subset of a Banach space $E$. A mapping $T: K \rightarrow K$ is said to be Lipschitzian if for each $n \geq 1$ there exists a positive real number $k_{n}$ such that

$$
\left\|T^{n} x-T^{n} y\right\| \leq k_{n}\|x-y\|
$$

for all $x, y$ in $K$. A Lipschitzian mapping is said to be nonexpansive if $k_{n}=1$ for all $n \geq 1$ and asymptotically nonexpansive [5] if $\lim _{n \rightarrow \infty} k_{n}=1$.

A Lipschitzian mapping is said to be uniformly Lipschitzian if $k_{n}=k$ for all $n \geq 1$ or in other words, if the Lipschitz constant of $T^{n}$,

$$
\left\|\mid T^{n}\right\|=\sup \left\{\frac{\left\|T^{n} x-T^{n} y\right\|}{\|x-y\|}: x \neq y, x, y \in K\right\}<k, n \geq 1
$$

holds for some $k>0$.

Received September 18, 1997.

1991 Mathematics Subject Classification. 47H10.

Key words and phrases.p-uniformly convex Banach space, asymptotic center, generalized Lipschitzian mapping, fixed point. 
In [8], Lifshitz proved the following result:

Theorem A. Let $K$ be a nonempty closed convex bounded subset of a Hilbert space. If $T: K \rightarrow K$ is a mapping such that

$$
\limsup _{n \rightarrow \infty}\left\|\left|T^{n} \|\right|<\sqrt{2}\right.
$$

then $T$ has a fixed point in $K$.

Let $p>1$ and denote by $\lambda$ a number in $[0,1]$ and by $\omega_{p}(\lambda)$ the function $\lambda \cdot(1-\lambda)^{p}+$ $\lambda^{p} \cdot(1-\lambda)$. The functional $\|\cdot\|^{p}$ is said to be uniformly convex (c. f. Zalinescu [16]) on the Banach space $E$ if there exists a positive constant $c_{p}$ such that for all $\lambda \in[0,1]$ and $x, y \in E$ the following inequality holds:

$$
\left\|\lambda_{x}+(1-\lambda) y\right\|^{p} \leq \lambda\|x\|^{p}+(1-\lambda)\|y\|^{p}-c_{p} \cdot \omega_{p}(\lambda) \cdot\|x-y\|^{p}
$$

$\mathrm{Xu}$ [15] proved that the functional $\|\cdot\|^{p}$ is uniformly convex on the whole Banach space $E$ if and only if $E$ is $p$-uniformly convex, i.e., there exists a constant $c_{p}>0$ such that the moduli of convexity, $\delta_{E}(\varepsilon) \geq c_{p} \cdot \varepsilon^{p}$ for all $0 \leq \varepsilon \leq 2$.

Górnicki and Krüppel [7] extended Theorem $\bar{A}$ via an inequality in Banach space and proved the following:

Theorem B. Let $E$ be a uniformly convex Banach space with diam $E>2$ for which norm satisfies (1) for some $p \geq 2$ and let $K$ be a nonempty bounded closed convex subset of $E$. If $T: K \rightarrow K$ is a mapping such that

$$
\lim _{n \rightarrow \infty} \frac{1}{n} \sum_{i=1}^{n}\left\|\mid T^{i}\right\|^{p}<1+c_{p}
$$

then $T$ has a fixed point in $K$.

Recently, Górnicki [6] generalized Theorem B to the following result via Banach space inequalities and more general summation methods involving

$$
\sum_{k=1}^{\infty} t_{n, k} \cdot\left\|\mid T^{k}\right\|^{p}, \quad n=1,2, \ldots
$$

where $A=\left[t_{n, k}\right]_{n, k \geq 1}$ is strongly ergodic matrix [2]:

(a) $\Lambda_{n, k} t_{n, k} \geq 0$,

(b) $\Lambda_{k} \lim _{n \rightarrow \infty} t_{n, k}=0$,

(c) $\Lambda_{n} \sum_{k=1}^{\infty} t_{n, k}=1$,

(d) $\lim _{n \rightarrow \infty} \sum_{k=1}^{\infty}\left|t_{n, k+1}-t_{n, k}\right|=0$

Theorem C. Let $p>1$ and let $E$ be a p-uniformly convex Banach space, $K a$ nonempty bounded closed convex subset of $E$, and $A=\left[t_{n, k}\right]_{n, k \geq 1}$ a strongly ergodic matrix. If $T: K \rightarrow K$ is a mapping such that

$$
g=\liminf _{n \rightarrow \infty} \inf _{m=0,1,2, \ldots} \sum_{k=1}^{\infty} t_{n, k}\left\|\left|T^{k+m} \|\right|^{p}<1+c_{p}\right.
$$


then $T$ has a fixed point in $K$.

We now consider the following class of mappings which we call generalized Lipschitzian mapping:

A mapping $T: K \rightarrow K$ is said to be generalized Lipschitzian if

$$
\begin{aligned}
& \left\|T^{n} x-T^{n} y\right\| \\
\leq & a_{n}\|x-y\|+b_{n}\left(\left\|x-T^{n} x\right\|+\left\|y-T^{n} y\right\|\right)+c_{n}\left(\left\|x-T^{n} y\right\|+\left\|y-T^{n} x\right\|\right)
\end{aligned}
$$

for each $x, y$ in $K$ and $n \geq 1$, where $a_{n}, b_{n}, c_{n}$ are nonnegative constants such that $3\left(b_{n}+c_{n}\right) \leq 1$ for all $n \geq 1$.

This class of mappings are more general than nonexpansive, asymptotically nonespansive, Lipschitzian and uniformly Lipschitzian mappings and it can be seen by taking $b_{n}=c_{n}=0$.

In the present paper, we extend the result of Górnicki [6] and consequently Górnicki and Krüppel [7] and Lipschitz [8] for generalized Lipschitzian mappings and in $p$-uniformly convex Banach space.Further, we establish for these mappings some fixed point theorems in Hilbert space, $L^{p}$ spaces, Hardy space $H^{p}$ or Sobolev spaces $H^{k, p}$ for $1<p<\infty$ and $k \geq 0$.

\section{Main Results}

Before presenting our main result, we need the following:

Lemma 1 [6]. Let $p>1$ and let $E$ be a p-uniformly convex Banach space, $K$ a nonempty closed convex subset of $E$, and $\left\{x_{n}\right\} \subset E$ a bounded sequence. Then there exists a unique point $z$ in $K$ such that

$$
\begin{aligned}
& \limsup _{n \rightarrow \infty} \sum_{k=1}^{\infty} t_{n, k} \cdot\left\|x_{k}-z\right\|^{p} \\
\leq & \limsup _{n \rightarrow \infty} \sum_{k=1}^{\infty} t_{n, k} \cdot\left\|x_{k}-x\right\|^{p}-c_{p} \cdot\|x-z\|^{p}
\end{aligned}
$$

for every $x$ in $K$, where $c_{p}$ is the constant given in (1) and $A=\left[t_{n, k}\right]_{n, k \geq 1}$ is a strongly ergodic matrix.

We are now in position to give our result:

Theorem 1. Let $p>1$ and let $E$ be a p-uniformly convex Banach space, $K a$ nonempty bounded closed convex subset of $E$, and $A=\left[t_{n, k}\right]_{n, k \geq 1}$ a strongly ergodic matrix. If $T: K \rightarrow K$ is a continuous generalized Lipschitzian mapping such that

$$
h=\liminf _{n \rightarrow \infty} \inf _{m=0,1,2, \ldots} \sum_{k=1}^{\infty} t_{n, k} \cdot 2^{p-1}\left\{\alpha_{k+m}^{p}+\beta_{k+m}^{p}\right\}<1+c_{p},
$$


where

and

$$
\alpha_{k+m}=\frac{a_{k+m}+b_{k+m}+c_{k+m}}{1-b_{k+m}-c_{k+m}}
$$

then $T$ has a fixed point in $K$.

$$
\beta_{k+m}=\frac{2 b_{k+m}+2 c_{k+m}}{1-b_{k+m}-c_{k+m}}
$$

Proof. Let $\left\{n_{i}\right\}$ and $\left\{m_{i}\right\}$ be sequences of natural numbers such that

$$
h=\liminf _{i \rightarrow \infty} \inf _{m=0,1,2, \ldots} \sum_{k=1}^{\infty} t_{n_{i}, k} \cdot 2^{p-1}\left\{\alpha_{k+m_{i}}^{p}+\beta_{k+m_{i}}^{p}\right\}<1+c_{p} .
$$

For any $z_{0} \in K$, we can inductively define a sequence $\left\{z_{j}\right\}$ in the following manner: $z_{j}$ is the unique asymptotic center in $K$ of the sequence $\left\{T^{n} z_{j-1}\right\}_{n \geq 1}$, i.e., $z_{j}$ is the unique point in $K$ that minimizes the functional

$$
r_{j-1}(x)=\limsup _{i \rightarrow \infty} \sum_{k=1}^{\infty} t_{n_{i}, k} \cdot\left\|x-T^{k+m_{i}} z_{j-1}\right\|^{p}
$$

over $x$ in $K$. Using (2), after a simple calculation, for each $x, y \in K, k>N \geq 1$, we have

$$
\begin{aligned}
& \left\|T^{N} x-T^{k} y\right\| \\
& \quad \leq \frac{a_{N}+b_{N}+c_{N}}{1-b_{N}-c_{N}} \cdot\left\|x-T^{k-N} y\right\|+\frac{2 b_{N}+2 c_{N}}{1-b_{N}-c_{N}} \cdot\left\|x-T^{k} y\right\| \\
& =\alpha_{N} \cdot\left\|x-T^{k-N} y\right\|+\beta_{N} \cdot\left\|x-T^{k} y\right\|
\end{aligned}
$$

In view of inequality (1), for any fixed $N, k, m_{i} \in \mathbb{N}$ and $0 \leq \lambda \leq 1$, we have

$$
\begin{aligned}
& \left\|\lambda z_{j}+(1-\lambda) \cdot T^{N} z_{j}-T^{k+m_{i}} z_{j-1}\right\|^{p} \\
& =\left\|\lambda\left(z_{j}-T^{k+m_{i}} z_{j-1}\right)+(1-\lambda)\left(T^{N} z_{j}-T^{k+m_{i}} z_{j-1}\right)\right\|^{p} \\
& \leq \lambda \cdot\left\|z_{j}-T^{k+m_{i}} z_{j-1}\right\|^{p}+(1-\lambda) \cdot\left\|T^{N} z_{j}-T^{k+m_{i}} z_{j-1}\right\|^{p} \\
& -c_{p} \cdot \omega_{p}(\lambda) \cdot\left\|z_{j}-T^{N} z_{j}\right\|^{p} .
\end{aligned}
$$

Multiplying both sides of this inequality by suitable elements of the matrix $A$ and summing, we have

$$
\begin{aligned}
& \sum_{k=1}^{\infty} t_{n_{i}, k} \cdot\left\|\lambda z_{j}+(1-\lambda) T^{N} z_{j}-T^{k+m_{i}} z_{j-1}\right\|^{p} \\
& \leq \lambda \cdot \sum_{k=1}^{\infty} t_{n_{i}, k} \cdot\left\|z_{j}-T^{k+m_{i}} z_{j-1}\right\|^{p} \\
& \quad+(1-\lambda) \cdot \sum_{k=1}^{\infty} t_{n_{i}, k} \cdot\left\|T^{N} z_{j}-T^{k+m_{i}} z_{j-1}\right\|^{P} \\
& \quad-c_{p} \cdot \omega_{p}(\lambda) \cdot\left\|z_{j}-T^{N} z_{j}\right\|^{p} \quad \text { for } i=1,2, \ldots
\end{aligned}
$$


Taking the limit superior on each side as $i \rightarrow \infty$, we obtain

$$
\begin{aligned}
& \limsup _{i \rightarrow \infty} \sum_{k=1}^{\infty} t_{n_{i}, k}\left\|\lambda z_{j}+(1-\lambda) T^{N} z_{j}-T^{k+m_{i}} z_{j-1}\right\|^{p} \\
& \leq \lambda \cdot \limsup _{i \rightarrow \infty} \sum_{k=1}^{\infty} t_{n_{i}, k} \cdot\left\|z_{j}-T^{k+m_{i}} z_{j-1}\right\|^{p} \\
& \quad+(1-\lambda) \cdot \limsup _{i \rightarrow \infty} \sum_{k=1}^{\infty} t_{n_{i}, k} \cdot\left\|T^{N} z_{j}-T^{k+m_{i}} z_{j-1}\right\|^{P} \\
& \quad-c_{p} \cdot \omega_{p}(\lambda) \cdot\left\|z_{j}-T^{N} z_{j}\right\|^{p}
\end{aligned}
$$

and

$$
\begin{aligned}
& c_{p} \cdot \omega_{p}(\lambda) \cdot\left\|z_{j}-T^{N} z_{j}\right\|^{p} \\
& \leq \lambda \cdot \underset{i \rightarrow \infty}{\limsup } \sum_{k=1}^{\infty} t_{n_{i}, k} \cdot\left\|z_{j}-T^{k+m_{i}} z_{j-1}\right\|^{p} \\
& \quad+(1-\lambda) \limsup _{i \rightarrow \infty} \sum_{k=1}^{\infty} t_{n_{i}, k} \cdot\left\|T^{N} z_{j}-T^{k+m_{i}} z_{j-1}\right\|^{p} \\
& \quad-\underset{i \rightarrow \infty}{\limsup } \sum_{k=1}^{\infty} t_{n_{i}, k} \cdot\left\|\lambda z_{j}+(1-\lambda) T^{N} z_{j}-T^{k+m_{i}} z_{j-1}\right\|^{p} \\
& \leq(1-\lambda) \cdot \limsup _{i \rightarrow \infty} \sum_{k=1}^{\infty} t_{n_{i}, k} \cdot\left\|T^{N} z_{j}-T^{k+m_{i}} z_{j-1}\right\|^{p} \\
& \quad-(1-\lambda) \cdot \limsup _{i \rightarrow \infty} \sum_{k=1}^{\infty} t_{n_{i}, k} \cdot\left\|z_{j}-T^{k+m_{i}} z_{j-1}\right\|^{p}
\end{aligned}
$$

and

$$
\begin{aligned}
& c_{p} \cdot\left[\lambda(\mathbb{1}-\lambda)^{p-1}+\lambda^{p}\right] \cdot\left\|z_{j}-T^{N} z_{j}\right\|^{p} \\
& \leq \limsup _{i \rightarrow \infty} \sum_{k=1}^{\infty} t_{n_{i}, k} \cdot\left\|T^{N} z_{j}-T^{k+m_{i}} z_{j-1}\right\|^{p} \\
& \quad-\limsup _{i \rightarrow \infty} \sum_{k=1}^{\infty} t_{n_{i}, k} \cdot\left\|z_{j}-T^{k+m_{i}} z_{j-1}\right\|^{p} .
\end{aligned}
$$

Taking $\lambda=1$, we get

$$
\begin{aligned}
c_{p} \cdot\left\|z_{j}-T^{N} z_{j}\right\|^{p} \leq & \limsup _{i \rightarrow \infty} \sum_{k=1}^{\infty} t_{n_{i}, k} \cdot\left\|T^{N} z_{j}-T^{k+m_{i}} z_{j-1}\right\|^{p} \\
& \quad \underset{i \rightarrow \infty}{\limsup } \sum_{k=1}^{\infty} t_{n_{i}, k} \cdot\left\|z_{j}-T^{k+m_{i}} z_{j-1}\right\|^{p} .
\end{aligned}
$$


In view of inequality (4), we have

$$
\begin{aligned}
& c_{p} \cdot\left\|z_{j}-T^{N} z_{j}\right\|^{p} \\
& \leq \limsup _{i \rightarrow \infty}\left[\sum_{k=1}^{N} t_{n_{i}, k} \cdot\left\|T^{N} z_{j}-T^{k+m_{i}} z_{j-1}\right\|^{p}\right. \\
& \left.+\sum_{k=N+1}^{\infty} t_{n_{i}, k}\left\{\alpha N \cdot\left\|z_{j}-T^{k-N+m_{i}} z_{j-1}\right\|+\beta_{N} \cdot\left\|z_{j}-T^{k+m_{i}} z_{j-1}\right\|\right\}^{p}\right] \\
& -\underset{i \rightarrow \infty}{\limsup } \sum_{k=1}^{\infty} t_{n_{i}, k} \cdot\left\|z_{j}-T^{k+m_{i}} z_{j-1}\right\|^{p} \\
& \leq \limsup _{i \rightarrow \infty}\left[\sum_{k=N+1}^{N} t_{n_{i}, k} \cdot\left\|T^{N} z_{j}-T^{k+m_{i}} z_{j-1}\right\|^{p}\right. \\
& +2^{p-1}\left\{\alpha_{N}^{p} \sum_{k=N+1}^{\infty} t_{n_{i}, k} \cdot\left\|z_{j}-T^{k-N+m_{i}} z_{j-1}\right\|^{p}\right. \\
& \left.\left.+\beta_{N}^{p} \sum_{k=N+1}^{\infty} t_{n_{i}, k} \cdot\left\|z_{j}-T^{k+m_{i}} z_{j-1}\right\|^{p}\right\}\right] \\
& -\limsup _{i \rightarrow \infty} \sum_{k=1}^{\infty} t_{n_{i}, k} \cdot\left\|z_{j}-T^{k+m_{i}} z_{j-1}\right\|^{p} \\
& \leq \limsup _{i \rightarrow \infty}\left[\sum_{k=1}^{N} t_{n_{i}, k} \cdot\left\|T^{N} z_{j}-T^{k+m_{i}} z_{j-1}\right\|^{p}\right. \\
& +2^{p-1}\left\{\alpha_{N}^{p} \sum_{k=1}^{\infty} t_{n_{i}, k+N} \cdot\left\|z_{j}-T^{k+m_{i}} z_{j-1}\right\|^{p}\right. \\
& \left.\left.+\beta_{N}^{\dot{p}} \sum_{k=N+1}^{\infty} t_{n_{i}, k} \cdot\left\|z_{j}-T^{k+m_{i}} z_{j-1}\right\|^{p}\right\}\right] \\
& -\limsup _{i \rightarrow \infty} \sum_{k=1}^{\infty} t_{n_{i}, k} \cdot\left\|z_{j}-T^{k+m_{i}} z_{j-1}\right\|^{p} \\
& =\underset{i \rightarrow \infty}{\limsup }\left[\sum_{k=1}^{N} t_{n_{i}, k} \cdot\left\|T^{N} z_{j}-T^{k+m_{i}} z_{j-1}\right\|^{p}\right. \\
& +2^{p-1}\left\{\alpha _ { N } ^ { p } \cdot \left(\sum_{k=1}^{\infty} t_{n_{i}, k} \cdot\left\|z_{j}-T^{k+m_{i}} z_{j-1}\right\|^{p}\right.\right. \\
& \left.-\sum_{k=1}^{\infty}\left(t_{n_{1}, k}-t_{n_{i}, k+N}\right) \cdot\left\|z_{j}-T^{k+m_{i}} z_{j-1}\right\|^{p}\right) \\
& \left.\left.+\beta_{N}^{p}\left(\sum_{k=1}^{\infty} t_{n_{i}, k} \cdot\left\|z_{j}-T^{k+m_{i}} z_{j-1}\right\|^{p}-\sum_{k=1}^{N} t_{n_{i}, k}\left\|z_{j}-T^{k+m_{i}} z_{j-1}\right\|^{p}\right)\right\}\right]
\end{aligned}
$$




$$
\begin{aligned}
& -\limsup _{i \rightarrow \infty} \sum_{k=1}^{\infty} t_{n_{i}, k} \cdot\left\|z_{j}-T^{k+m_{i}} z_{j-1}\right\|^{p} \\
& \leq\left[2^{p-1}\left(\alpha_{N}^{p}+\beta_{N}^{p}\right)-1\right] \cdot \underset{i \rightarrow \infty}{\limsup } \sum_{k=1}^{\infty} t_{n_{i}, k} \cdot\left\|z_{j}-T^{k+m_{i}} z_{i-1}\right\|^{p}
\end{aligned}
$$

since

(i) $\sum_{k=1}^{N} t_{n_{i}, k} \cdot\left\|T^{N} z_{j}-T^{k+m_{i}} z_{j-1}\right\|^{p} \rightarrow 0$ as $i \rightarrow+\infty$,

(ii) $\sum_{k=1}^{\infty}\left(t_{n_{i}, k}-t_{n_{i}, k+N}\right) \cdot\left\|z_{j}-T^{k+m_{i}} z_{j-1}\right\|^{p} \rightarrow 0$ as $i \rightarrow+\infty$,

(iii) $r_{j-1}\left(z_{j}\right) \leq r_{j-1}\left(z_{j-1}\right)$.

For any fixed $N \in \mathbb{N}$, we have

$$
c_{p} \cdot\left\|z_{j}-T^{N} z_{j}\right\|^{p} \leq\left[2^{p-1}\left(\alpha_{N}^{p}+\beta_{N}^{p}\right)-1\right] \cdot r_{j-1}\left(z_{j-1}\right) .
$$

We multiply this inequality for $N=k+m_{i}$ by suitable elements $t_{n_{i}, k}$ for $k=1,2, \ldots$ Summing up these inequalities and taking the limit superior on each side as $i \rightarrow+\infty$, we obtain

$$
\begin{aligned}
& c_{p} \cdot \limsup _{i \rightarrow \infty} \sum_{k=1}^{\infty} t_{n_{i}, k} \cdot\left\|z_{j}-T^{k+m_{i}} z_{j}\right\|^{p} \\
& \quad \leq \lim _{i \rightarrow \infty} \sum_{k=1}^{\infty} t_{n_{i}, k} \cdot\left\{2^{p-1}\left(\alpha_{k+m_{i}}^{p}+\beta_{k+m_{i}}^{p}\right)-1\right\} \cdot r_{j-1}\left(z_{j-1}\right)
\end{aligned}
$$

and

$$
r_{j}\left(z_{j}\right) \leq B \cdot r_{j-1}\left(z_{j-1}\right)
$$

where

$$
B=\frac{1}{c_{p}}\left[\lim _{i \rightarrow \infty} \sum_{k=1}^{\infty} t_{n_{i}, k} \cdot\left\{2^{p-1}\left(\alpha_{k+m_{i}}^{p}+\beta_{k+m_{i}}^{p}\right)-1\right\}\right]<1 .
$$

In a similar way, we obtain

$$
r_{j}\left(z_{j}\right) \leq B^{j} \cdot r_{0}\left(z_{0}\right), \quad j=1,2, \ldots
$$

Next, we show the convergence of the sequence $\left\{z_{j}\right\}$. For a fixed $N \in \mathbb{N}$, we have

$$
\left\|z_{j+1}-z_{j}\right\|^{p} \leq 2^{p-1}\left(\left\|z_{j+1}-T^{N} z_{j}\right\|^{p}+\left\|T^{N} z_{j}-z_{j}\right\|^{p}\right)
$$

We multiply this inequality for $N=k+m_{i}$ by suitable elements $t_{n_{i}, k}$ for $k=1,2, \ldots$. Summing up these inequalities and taking the limit superior on each side as $i \rightarrow+\infty$, we obtain

$$
\begin{aligned}
\left\|z_{j+1}-z_{j}\right\|^{p} \leq & 2^{p-1}\left[\limsup _{i \rightarrow \infty} \sum_{k=1}^{\infty} t_{n_{i}, k} \cdot\left\|z_{j+1}-T^{N} z_{j}\right\|^{p}\right. \\
& \left.+\limsup _{i \rightarrow \infty} \sum_{k=1}^{\infty} t_{n_{i}, k} \cdot\left\|T^{N} z_{j}-z_{j}\right\|^{p}\right] \\
= & 2^{p-1} \cdot\left(r_{j}\left(z_{j+1}\right)+r_{j}\left(z_{j}\right)\right) \\
\leq & 2^{p} \cdot r_{j}\left(z_{j}\right) \\
\leq & 2^{p} \cdot B^{j} \cdot r_{0}\left(z_{0}\right)
\end{aligned}
$$


and

$$
\left\|z_{j+1}-z_{j}\right\| \leq 2\left[B^{j} \cdot r_{0}\left(z_{0}\right)\right]^{\frac{1}{p}} \rightarrow 0 \text { as } j \rightarrow+\infty,
$$

which show that $\left\{z_{j}\right\}$ is a Cauchy sequence. Let $z=\lim _{j \rightarrow \infty} z_{j}$. For fixed $N \in \mathbb{N}$, we have

$$
\begin{aligned}
& \left\|z-T^{N} z\right\| \\
& \leq\left\|z-z_{j}\right\|+\left\|z_{j}-T^{N} z_{j}\right\|+\left\|T^{N} z_{j}-T^{N} z\right\| \\
& \leq \frac{1+a_{N}+2 c_{N}}{1-b_{N}-c_{N}} \cdot\left\|z-z_{j}\right\|+\frac{1+b_{N}-c_{N}}{1-b_{N}-c_{N}} \cdot\left\|z_{j}-T^{N} z_{j}\right\|
\end{aligned}
$$

or

$$
\begin{aligned}
& \left\|z-T^{N} z\right\|^{p} \\
& \leq 2^{p-1} \cdot\left[\left(\frac{1+a_{N}+2 c_{N}}{1-b_{N}-c_{N}}\right)^{p} \cdot\left\|z-z_{j}\right\|^{p}+\left(\frac{1+b_{N}+c_{N}}{1-b_{N}-c_{n}}\right)^{p} \cdot\left\|z_{j}-T^{N} z_{j}\right\|^{p}\right] \\
& \leq 2^{p-1} \cdot\left[\left(\frac{1+a_{N}+2 c_{N}}{1-b_{N}-c_{N}}\right)^{p} \cdot\left\|z-z_{j}\right\|^{p}+2^{p} \cdot\left\|z_{j}-T^{N} z_{j}\right\|^{p}\right] .
\end{aligned}
$$

We multiply this inequality for $N=k+m_{i}$ by suitable elements $t_{n_{i}, k}$ for $k=1,2, \ldots$ Summing up these inequalities, we obtain

$$
\begin{aligned}
& \sum_{k=1}^{\infty} t_{n_{i}, k} \cdot\left\|z-T^{k+m_{i}} z\right\|^{p} \\
& \leq 2^{p-1}\left[\left\|z-z_{j}\right\|^{p} \cdot \sum_{k=1}^{\infty} t_{n_{i}, k} \cdot\left(\frac{1+a_{k+m_{i}}+2 c_{k+m_{i}}}{1-b_{k+m_{i}}-c_{k+m_{i}}}\right)^{p}\right. \\
& \left.\quad+2^{p} \cdot \sum_{k=1}^{\infty} t_{n_{i}, k}\left\|z_{j}-T^{k+m_{i}} z_{j}\right\|^{p}\right] .
\end{aligned}
$$

Taking the limit superior on each side as $i \rightarrow+\infty$, we get

$$
\begin{aligned}
& \limsup _{i \rightarrow \infty} \sum_{k=1}^{\infty} t_{n_{i}, k} \cdot\left\|z-T^{k+m_{i}} z\right\|^{p} \\
& \leq 2^{p-1} \cdot\left[\left\|z-z_{j}\right\|^{p} \cdot \limsup _{i \rightarrow \infty} \sum_{k=1}^{\infty} t_{n_{i}, k} \cdot\left(\frac{1+a_{k+m_{i}}+2 c_{k+m_{i}}}{1-b_{k+m_{i}}-c_{k+m_{i}}}\right)^{p}\right. \\
& \left.\quad+2^{p} \cdot \limsup _{i \rightarrow \infty} \sum_{k=1}^{\infty} t_{n_{i}, k}\left\|z_{j}-T^{k+m_{i}} z_{j}\right\|^{p}\right] \\
& \leq 2^{p-1} \cdot\left[\left\|z-z_{j}\right\|^{p} \cdot \limsup _{i \rightarrow \infty} \sum_{k=1}^{\infty} t_{n_{i}, k} \cdot\left(\frac{1+a_{k+m_{i}}+2 c_{k+m_{i}}}{1-b_{k+m_{i}}-c_{k+m_{i}}}\right)^{p}+2^{p} \cdot B^{j} r_{0}\left(z_{0}\right)\right] \\
& \rightarrow 0 \text { as } j \rightarrow+\infty
\end{aligned}
$$


Therefore,

$$
\limsup _{i \rightarrow \infty} \sum_{k=1}^{\infty} t_{n_{i}, k} \cdot\left\|z-T^{k+m_{i}} z\right\|^{p}=0
$$

This implies that $T_{z}=z$. Indeed, for any $\varepsilon>0$ there exists natural numbers $n, n+1$ such that

$$
\left\|z-T^{n} z\right\|<\varepsilon \text { and }\left\|z-T^{n+1} z\right\|<\varepsilon .
$$

Otherwise, we have for any $n$ and $m$.

$$
\sum_{k=1}^{\infty} t_{n, k} \cdot\left\|z-T^{k+m} z\right\|^{p} \geq \frac{1}{2} \varepsilon^{p}
$$

and hence

$$
\limsup _{i \rightarrow \infty} \sum_{k=1}^{\infty} t_{n_{i}, k} \cdot\left\|z-T^{k+m_{i}} z\right\|^{p} \geq \underset{i \rightarrow \infty}{\limsup } \sum_{k=1}^{\infty} t_{n_{i}, k} \cdot\left\|z-T^{k+m_{i}} z\right\|^{p} \geq \frac{1}{2} \varepsilon^{p} .
$$

Thus for every natural number $l$ there exists a natural number $n_{1}$ such that

$$
\left\|z-T^{n_{l}} z\right\|<\frac{1}{l} \text { and }\left\|z-T^{n_{l}+1} z\right\|<\frac{1}{l} .
$$

It follows that

$$
T^{n_{l}} z \rightarrow z \text { and } T^{n_{l}+1} \rightarrow z \text { as } l \rightarrow \infty .
$$

Since $T$ is continuous, we have

$$
T_{z}=T\left(\lim _{l \rightarrow \infty} T^{n_{l}} z\right)=\lim _{l \rightarrow \infty} T^{n_{l}+1} z=z .
$$

This completes the proof.

\section{Some Applications}

In a Hilbert space $H$, the following identity holds:

$$
\|\lambda x+(1-\lambda) y\|^{2}=\lambda\|x\|^{2}+(1-\lambda)\|y\|^{2}-\lambda(1-\lambda)\|x-y\|^{2}
$$

for all $x, y$ in $H$ and $\lambda \in[0,1]$.

By (5), we immediately obtain from Theorem 1 the following:

Corollary 1. Let $K$ be a nonempty bounded closed convex subset of a Hilbert space $H$ and $A=\left[t_{n, k}\right]_{n, k \geq 1}$ a strongly ergodic matrix. If $T: K \rightarrow K$ is a continuous generalized Lipschitzian mapping such that

$$
\liminf _{i \rightarrow \infty} \inf _{m=0,1,2, \ldots} \sum_{k=1}^{\infty} t_{n, k} \cdot\left(\alpha_{n+m}^{2}+\beta_{k+m}^{2}\right)<1,
$$


then $T$ has a fixed point in $K$.

If $1<p \leq 2$, then we have for all $x, y$ in $L^{P}$ and $\lambda \in[0,1]$

$$
\|\lambda x+(1-\lambda) y\|^{2} \leq \lambda\|x\|^{2}+(1-\lambda)\|y\|^{2}-\lambda(1-\lambda) \cdot(p-1)\|x-y\|^{2}
$$

(The inequality (6) is contained in $\mathrm{Lim}, \mathrm{Xu}$ and $\mathrm{Xu}$ [10] and Smarzewski [14].)

Assume that $2<p<+\infty$ and $h_{p}$ is the unique zero of the function $g(x)=-x^{p-1}+$ $(p-1) x+p-2$ in the interval $(1, \infty)$. Let

$$
c_{p}=(p-1) \cdot\left(1+h_{p}\right)^{2-p}=\frac{1+h_{p}^{p-1}}{\left(1+h_{p}\right)^{p-1}} .
$$

Then we have the following inequality:

$$
\|\lambda x+(1-\lambda) y\|^{p} \leq \lambda\|x\|^{p}+(1-\lambda)\|y\|^{p}=\omega_{p}(\lambda) \cdot c_{p} \cdot\|x-y\|^{p}
$$

for all $x, y$ in $L^{p}$ and $\lambda \in[0,1]$ (The inequality (7) is essentially due to Lim [9]).

Corollary 2. Let $K$ be a nonempty bounded closed convex subset of $L^{p}(1<p<+\infty)$ and $A=\left[t_{n, k}\right]_{n, k \geq 1}$ a strongly ergodic matrix. If $T: K \rightarrow K$ is a continuous generalized Lipschitzian mapping such that

$$
\liminf _{n \rightarrow \infty} \inf _{m=0,1,2, \ldots} \sum_{k=1}^{\infty} t_{n, k} \cdot 2\left(\alpha_{k+m}^{2}+\beta_{k+m}^{2}\right)<p, \text { for } 1<p \leq 2
$$

and

$$
\liminf _{n \rightarrow \infty} \inf _{m=0,1,2, \ldots} \sum_{k=1}^{\infty} t_{n, k} \cdot 2^{p-1}\left(\alpha_{k+m}^{p}+\beta_{k+m}^{p}\right)<1+c_{p}, \quad \text { for } p>2,
$$

Then $T$ has a fixed point in $K$.

Let $H^{p}, 1<p<+\infty$, denote the Hardy space [4] of all functions $x$ analytic in unit disc $|z|<1$ of the complex plane and such that

$$
\|x\|=\lim _{r \rightarrow 1^{-}}\left(\frac{1}{2 \pi} \int_{0}^{2 \pi}\left|x\left(r e^{i \theta}\right)\right|^{p} d \theta\right)^{\frac{1}{p}}<\infty
$$

Now, let $\Omega$ be an open subset of $\mathbb{R}^{n}$. Denote by $H^{k, p}(\Omega), k \geq 0,1<p<+\infty$, the Sobolev space $\left[1\right.$, p. 149] of distributions $x$ such that $D^{\alpha} x \in L^{p}(\Omega)$ for all $|\alpha|=$ $\alpha_{1}+\cdots+\alpha_{n} \leq k$ equipped with the norm.

$$
\|x\|=\left(\sum_{|\alpha| \leq k} \int_{\Omega}\left|D^{\alpha} x(\omega)\right|^{p} d \omega\right)^{\frac{1}{p}} .
$$

Let $\left(\Omega_{\alpha}, \sum_{\alpha}, \mu_{\alpha}\right), \alpha \in \Lambda$, be a sequence of positive measure spaces, where index set $\Lambda$ is finite or countable. Given a sequence of linear subspaces $X_{\alpha}$ in $L^{p}\left(\Omega_{\alpha}, \Sigma_{\alpha}\right.$, $\left.\mu_{\alpha}\right)$, we 
denote by $L_{q, p}, 1<p<+\infty$ and $q=\max \{2, p\}$ [11], the linear space of all sequence $x\left\{x_{\alpha} \in X_{\alpha}: \alpha \in \Lambda\right\}$ equipped with the norm

$$
\|x\|=\left(\sum_{\alpha \in \Lambda}\left(\left\|x_{\alpha}\right\|_{p, \alpha}\right)^{q}\right)^{\frac{1}{\varepsilon}} .
$$

where $\|\cdot\|_{p, \alpha}$ denotes the norm in $L^{p}\left(\Omega_{\alpha}, \sum_{\alpha}, \mu_{\alpha}\right)$.

Finally, let $L_{p}=L^{p}\left(S_{1}, \sum_{1}, \mu_{1}\right)$ and $L_{q}=L^{q}\left(S_{2}, \sum_{2}, \mu_{2}\right)$, where $1<p<+\infty$, $q=\max \{2, p\}$ and $\left(S_{i}, \sum_{i}, \mu_{i}\right)$ are positive measure spaces. Denote by $L_{q}\left(L_{p}\right)$ the Banach spaces [3, III. 2.10] of all measureble $L_{p}$-value function $x$ on $S_{2}$ such that

$$
\|x\|=\left(\int_{S_{2}}\left(\|x(s)\|_{p}\right)^{q} \mu_{2}(d s)\right)^{\frac{1}{\varepsilon}} .
$$

These spaces are $q$-uniformly convex with $q=\max \{2, p\}[12,13]$ and the norm in these spaces satisfies

$$
\|\lambda x+(1-\lambda) y\|^{q} \leq \lambda\|x\|^{q}+(1-\lambda)\|y\|^{q}-d \cdot \omega_{q}(\lambda) \cdot\|x-y\|^{q}
$$

with a constant

$$
d=d_{p}=\left\{\begin{array}{lc}
\frac{p-1}{8} \quad \text { for } 1<p \leq 2 \\
\frac{1}{p \cdot 2^{p}} \text { for } 2<p<+\infty
\end{array}\right.
$$

Here, from Theorem 1, we have the following result:

Corollary 3. Let $K$ be a nonempty bounded closed convex subset of the space $E$, where $E=H^{p}$, or $E=H^{k, p}(\Omega)$, or $E=L_{q, p}$, or $E=L_{q}\left(L_{p}\right)$, and $1<p<+\infty$, $q=\max \{2, p\}, k \geq 0$ and $A=\left[t_{n, k}\right]_{n, k \geq 1}$ is a strongly ergodic matrix. If $T: K \rightarrow K$ is a continuous generalized Lipschitzian mapping such that

$$
\liminf _{n \rightarrow \infty} \inf _{m=0,1,2, \ldots} \sum_{k=1}^{\infty} t_{n, k} \cdot 2^{q-1}\left\{\alpha_{k+m}^{q}+\beta_{k+m}^{q}\right\}<1+d_{p},
$$

then $T$ has a fixed point in $K$.

\section{Acknowledgement}

The third author was supported by the Basic Science Research Institute Program, Ministry of Education, Korea, 1997, Project No. BSRI-97-1405.

\section{References}

[1] J. Barros-Neto, "An Introduction to the theory of distribution," Dekker, New York, 1973.

[2] R. E. Bruck, "On the almost-convergence of iterates of a nonexpansive mapping in Hilbert space and the structure of weak $\omega$-limit set," Israel J. Math., 29(1978), 1-6. 
[3] N. Dunford and J. Schwarz, Linear Operators, Vol. I. Interscience, New York, 1958.

[4] W. L. Duren, Theory of $H^{p}$ Spaces, Academic Press, New York., 1970.

[5] K. Goebel and W. A. Kirk, "A fixed point theorem for asymptotically nonexpansive mappings," Proc. Amer. Math. Soc., 35(1972), 171-174.

[6] J. Górnicki, "A remark on fixed point theorems for Lipschitzian mappings," J. Math. Anal. Appl., 183(1994), 495-508.

[7] J. Górnicki and M. Krüppel, "Fixed point theorems for mappings with Lipschizian iterates," Nonlinear Anal. TMA., 19(4) (1992), 353-363.

[8] E. A. Lifshitz, "Fixed point theorem for operators in strongly convex spaces," Voronez Gos. Univ. Trudy Math. Fak., 16(1975), 23-38 (in Russian).

[9] Lim, T. C., "On some $L^{p}$ inequalities in best approxination theory," J. Math. Anal. Appl., 154(1991), 523-528.

[10] T. C. Lim, H. K. Xu and Z. B. Xu, "An $L^{p}$ inequalities and its applications to fixed point theory and approximation theory," Progress in Approximation Theory, Academic Press, 1991, 609-624.

[11] J. Lindenstrauss and L. Tzafriri, "Classical Banach spaces II-Function Spaces," SpringerVerlag, New York, Berlin, 1979.

[12] B. Prus and R. Smarzewski, "Strongly unique best approximations and centers in uniformly convex spaces," J. Math. Anal. Appl., 121(1987), 10-21.

[13] R. Smarzewski, "Strongly unique best approximations in Banach spaces II," J. Approx. Theory, 51(1987), 202-217.

[14] R. Smarzewski, "On the inequality of Bynum and Drew," J. Math. Anal. Appl., 150(1990), 146-150.

[15] H. K. Xu, "Inequalities in Banach spaces with applications," Nonlinear Anal. TMA., 16(1991), 1127-1138.

[16] C. Zalinescu, "On uniformly convex function," J. Math. Anal. Appl., 95(1983), 344-374.

Govt. B. H. S. S. Gariaband, Dist. Raipur, M. P. 493889, India.

School of Studies in Mathematics, Pt. Ravishankar Shukla, University, Raipur 492010, India.

Department of Mathematics, Dong-A University, Pusan 607-714, Korea.

(E-mail: jungjs@seunghak.donga.ac.kr) 